\title{
Croissance de l'érable à sucre et du hêtre à grandes feuilles selon la position sociale dans le peuplement
}

\author{
R. ZARNOVICAN, J. TRENCIA * \\ C.F.L., Service canadien des fôrêts \\ 1055, rue du P.E.P.S., Sainte-Foy, Québec, Canada \\ * INRA, Centre de Recherches de Nancy, \\ Station de Sylviculture et de Production, Champenoux, F 54280 Seichamps
}

\begin{abstract}
Résumé
La reconstitution de la croissance et du développement de l'érable à sucre et du hêtre à grandes feuilles, appartenant à trois classes sociales différentes, a été effectuée à partir de l'analyse de 51 tiges.

D'après les résultats obtenus, la différenciation sociale des arbres dans le peuplement d'étude était déjà terminée à l'âge de trente ans, et la hiérarchie qui en a résulté était pratiquement fixée. Parmi les grandeurs dendrométriques du peuplement, on peut identifier le volume comme une grandeur très sensible et indicatrice de la position sociale de l'arbre dans le peuplement.
\end{abstract}

Mots clés : Acer saccharum, Fagus grandifolia, classes d'arbres, analyse de tiges.

\section{Introduction}

Un des traits dominants qui caractérise l'évolution des peuplements forestiers est sans doute la compétition entre les individus qui les composent. Cette lutte entre les arbres pour la place au soleil commence très tôt et la destinée d'un collectif d'arbres se joue en quelques années à partir de sa création (Assmann, 1961 ; Delvaux, 1964).

Le grégarisme du collectif d'arbres et la compétition dans un peuplement a son importance, car à l'opposé, l'absence totale de compétiton, dans le cas des arbres isolés, conduit d'abord à réduire la croissance, en hauteur en tout cas, et à une dépréciation non négligeable de la qualité du bois (SchütZ, 1981).

Le processus de la différenciation sociale et de la hiérarchisation entre les arbres, reflète l'existence d'individus qui, tout en étant de la même espèce, n'en possèdent pas moins des possibilités différentes et, comme le rappelle SchüTz (op. cit.), il existe des individus surdoués, comme il y a des ratés. Les arbres qui par hasard ou non se trouvent à dominer les autres à l'état de fourré vont conserver leur position dans le peuplement en repoussant ou écrasant les autres moins favorisés par le sort. 
A moins que le forestier n'intervienne. la hiérarchie sociale ne reflétera pas forcément le potentiel de croissance, et, à plus forte raison, la qualité des individus. Ceci est vrai spécialement chez les feuillus, où l'ensemble des opérations culturales commençant par les soins aux semis, ont une importance fondamentale dans la conduite des peuplements, car elles assurent le réglage des mélanges par la sélection des individus de qualité pour la meilleure composition de la strate dominante.

Notre travail se propose d'examiner la croissance et le développement de l'érable à sucre Acer saccharum Marsh. et du hêtre à grandes feuilles Fagus grandifolia Erh. provenant d'un haut perchis et appartenant, lors de l'abattage, aux trois principales classes sociales. Cet examen a pour but de déterminer le processus de différenciation des arbres en classes sociales dans le temps et. leur croissance en différentes grandeurs dendrométriques.

Mentionnons, qu'au plan commercial, l’érable à sucre se situe parmi les plus importantes essences commerciales du Canada et ses utilisations industrielles sont nombreuses. Quant au hêtre à grandes feuilles, c'est une essence secondaire avec une utilisation industrielle restreinte.

La présente étude a été effectuée dans le massif boisé de Lejeune, situé à $47^{\circ} 44^{\prime}$ de latitude nord et à $68^{\circ} 34^{\prime}$ de longitude ouest, dans le comté de Témiscouata à $350 \mathrm{~m}$ d'altitude, province de Québec, Canada. Le climat témiscouatain est continental tempéré (Villeneuve, 1959) avec une période de croissance annuelle moyenne de 170 jours, une moyenne annuelle des précipitations totales de $1000 \mathrm{~mm}$ et une température annuelle moyenne de $2,5^{\circ} \mathrm{C}$ (WILSON, 1971).

La région immédiate du massif boisé fait partie du domaine climacique de l'érablière à bouleau jaune de GrandTNER (1966), le groupement caractéristique des stations mésiques. D'après Rowe (1972), le massif se situe dans la région forestière des Grands Lacs et du St-Laurent, section L6 - Témiscouata - Restigouche.

Enfin, les sols du massif sont peu profonds, pierreux, bien drainés et formés de résidus de schiste ardoisier à texture limoneuse. Le massif est situé sur un moyen versant de pente régulière de 8 p. 100 et son exposition est vers le sud-ouest.

\section{Matériel et méthodes}

Les données sur la croissance et le développement des deux essences proviennent d'analyse de tige, dont la description méthodologique a été donnée dans un travail récent (ZaRNovicaN, 1985). L'abattage des arbres a été réalisé lors d'une éclaircie expérimentale en 1984 sur une superficie de 0,5 ha et au total, 33 hêtres à grandes feuilles et 28 érables à sucre ont été échantillonnés, en utilisant des longueurs de section de $1 \mathrm{~m}$. Le choix d'arbres sur le terrain a été réalisé de manière à avoir approximativement le même nombre d'arbres par strate verticale reconnaissable dans le peuplement, à savoir, la strate supérieure, moyenne et inférieure. Nous avons éliminé de notre échantillonnage les arbres portant des défauts visibles sur la tige et sur la cime.

Le peuplement d'étude provient d'une régénération naturelle après coupe à blanc et il est actuellement constitué par trois essences principales : l'érable à sucre, le hêtre 
à grandes feuilles et le bouleau jaune (Betula alleghaniensis Britton). Mais, sur la surface traitée, l'érable est l'essence dominante et il est accompagné du hêtre à grandes feuilles. ment.

A notre connaissance aucune opération culturale n'a été réalisée dans le peuple-

\section{Caractéristiques dendrométriques des arbres étudiés}

Avant l'abattage des arbres sur le terrain, nous avons noté leur appartenance à une des trois strates verticales et ceci, selon la hauteur de l'arbre. Cependant, le peuplement n'étant pas complètement homogène, il est possible que les moyennes des échantillons des classes diffèrent des moyennes de ces mêmes classes pour l'ensemble du peuplement. Les caractéristiques par classe d'arbres pour les deux essences sont rapportées au tableau 1 .

\section{TABleaU 1}

Caractéristiques dendrométriques des arbres analysés.

Characteristics of sampled trees.

\begin{tabular}{|c|c|c|c|c|c|c|c|c|c|c|}
\hline \multirow{2}{*}{ Essence } & \multirow{2}{*}{$\begin{array}{c}\text { Classe } \\
\text { d'arbres }\end{array}$} & \multirow{2}{*}{$\begin{array}{l}\text { Nombre } \\
\text { d'arbres }\end{array}$} & \multicolumn{2}{|c|}{$\begin{array}{c}\text { Age à la } \\
\text { souche (ans) }\end{array}$} & \multicolumn{2}{|c|}{$\begin{array}{l}\text { Hauteur } \\
\text { totale (m) }\end{array}$} & \multicolumn{2}{|c|}{$\begin{array}{l}\text { dhp } \\
(\mathrm{cm})\end{array}$} & \multicolumn{2}{|c|}{$\mathrm{h} / \mathrm{dhp}$} \\
\hline & & & moy. & sx & moy. & $s x$ & moy. & $\mathrm{sx}$ & moy. & $s x$ \\
\hline Erable à sucre & $\begin{array}{r}\text { I } \\
\text { II } \\
\text { III }\end{array}$ & $\begin{array}{r}10 \\
8 \\
10\end{array}$ & $\begin{array}{l}43 \\
42 \\
40\end{array}$ & $\begin{array}{l}2 \\
1 \\
3\end{array}$ & $\begin{array}{r}14,2 \\
11,9 \\
9,2\end{array}$ & $\begin{array}{l}0,7 \\
0,9 \\
0,6\end{array}$ & $\begin{array}{r}11,6 \\
7,7 \\
5,4\end{array}$ & $\begin{array}{l}1,1 \\
0,5 \\
0,6\end{array}$ & $\begin{array}{l}1,24 \\
1,56 \\
1,73\end{array}$ & $\begin{array}{l}0,13 \\
0,14 \\
0,13\end{array}$ \\
\hline $\begin{array}{l}\text { Hêtre à grandes } \\
\text { feuilles } \ldots \ldots \text {. }\end{array}$ & $\begin{array}{r}\text { I } \\
\text { II } \\
\text { III }\end{array}$ & $\begin{array}{r}11 \\
9 \\
13\end{array}$ & $\begin{array}{l}42 \\
42 \\
40\end{array}$ & $\begin{array}{l}1 \\
2 \\
2\end{array}$ & $\begin{array}{r}13,7 \\
11,7 \\
9,3\end{array}$ & $\begin{array}{l}0,9 \\
0,8 \\
1,2\end{array}$ & $\begin{array}{r}11,6 \\
8,7 \\
6,2\end{array}$ & $\begin{array}{l}1,0 \\
0,9 \\
0,8\end{array}$ & $\begin{array}{l}1,19 \\
1,35 \\
1,50\end{array}$ & $\begin{array}{l}0,15 \\
0,13 \\
0,17\end{array}$ \\
\hline
\end{tabular}

A l'examen du tableau, on note que les effectifs par classe d'arbres sont sensiblement les mêmes et ceci pour les deux essences. L'examen de l'âge des arbres indique une légère diminution de la première classe vers la troisième. Toutefois, cette diminution est relativement faible et au plan pratique, on peut considérer les arbres récoltés comme un ensemble équienne et faisant partie d'une même classe d'âge.

Cependant, c'est à l'examen des données sur la hauteur, le diamètre à hauteur d'homme (dhp) et le rapport $\mathrm{h} / \mathrm{dhp}$ du même tableau qu'il est possible de distinguer les classes d'arbres. Leur individualisation apparaît clairement à la figure 1. En effet, malgré la nature équienne de l'ensemble des arbres selon leur âge, l'existence de différentes classes définies par la hauteur et le diamètre à hauteur d'homme est évidente.

C'est sur la base de l'existence d'un ensemble équienne d'arbres des deux essences, appartenant aux trois classes sociales distinctes, que nous avons effectué l'étude sur la 

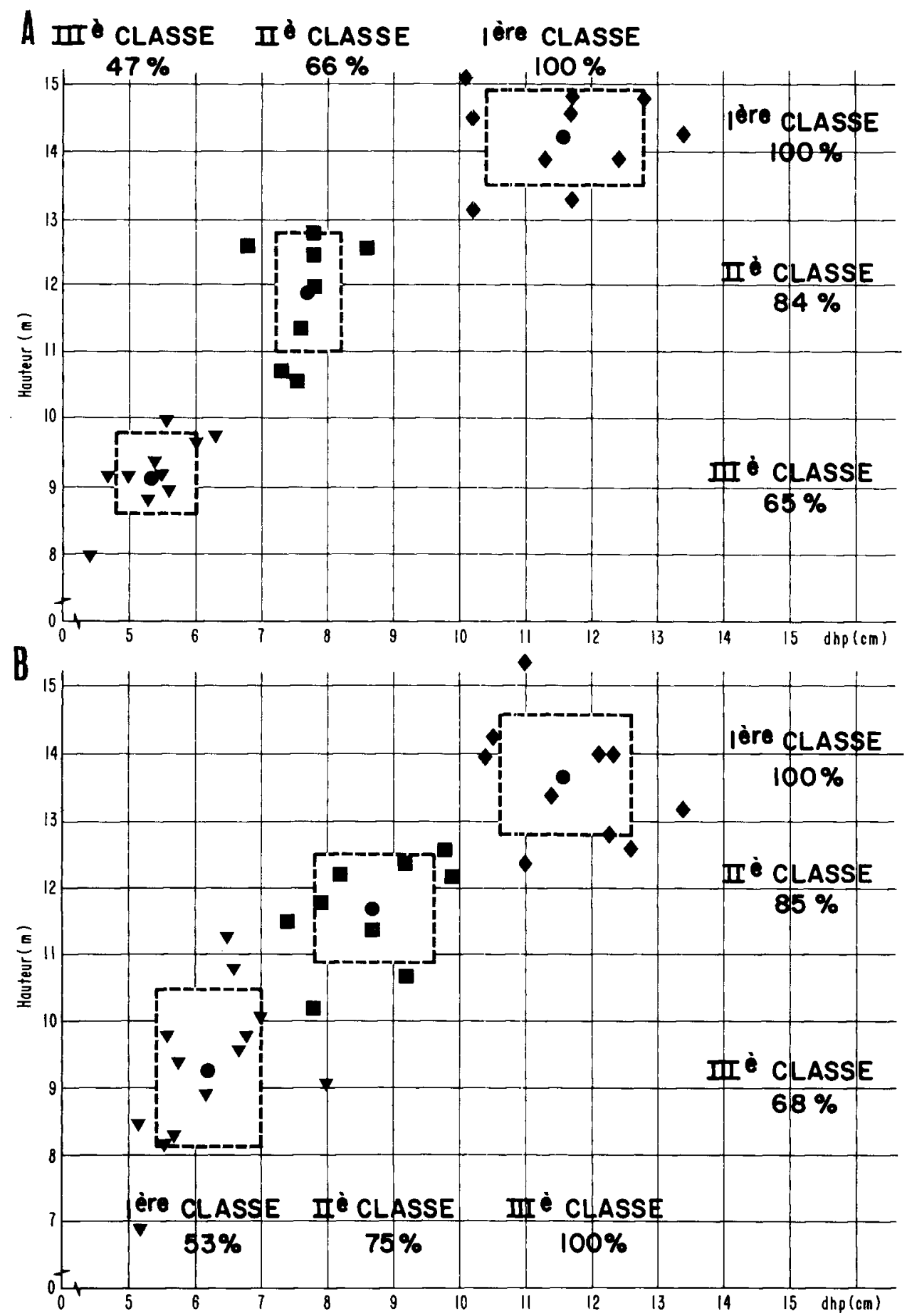

FIG. 1

Distribution de tiges d'érable à sucre $(A)$ et de hêtre à grandes feuilles $(B)$ selon le dhp et la hauteur de 1984.

(Les moyennes sont indiquées par des cercles et les écarts types par des pointillés).

Distribution of sugar maple $(A)$ and beech $(B)$ according to diameter and height in 1984.

Means are shown as circles and standard deviations as dashed lines. 
différenciation sociale et le développement en différentes grandeurs dendrométriques pour la période de 1960 à 1980 .

Cette rétrospective de la croissance et du développement des arbres selon la classe sociale nous paraît possible, car la position sociale des arbres est déjà fixée et selon les résultats d'autres travaux (SMElko, 1976; DvoreckiJ, 1966), la période de 20 ans permet une reconstruction précise de la croissance dans le passé.

Pour les besoins de la présente contribution, nous avons utilisé les données de l'analyse de tiges sur le diamètre, la hauteur, le volume (s.e.) et l'accroissement courant respectif qui sont établis par intervalle de 2 ans. A partir de ces données, nous avons calculé les intervalles de confiance au seuil de $95 \mathrm{p}$. 100 pour la moyenne de chaque variable dendrométrique étudiée ; ces intervalles de confiance sont indiqués sur les figures par des trames. Ils représentent une mesure objective des différences entre la croissance en dhp, en volume et en hauteur des principales classes d'arbres. On peut donc considérer les croissances comme significativement différentes dans les segments où les intervalles de confiance ne se recouvrent pas. Inversement, on peut considérer les différences comme statistiquement non significatives dans les segments où les intervalles se recouvrent.

Mentionnons de plus que les données sur la croissance en différentes grandeurs dendrométriques ne représentent pas la croissance d'un arbre réel, mais la croissance de l'arbre moyen, fictif, produit du calcul. Ce dernier change au cours de la vie d'un peuplement, dans sa grosseur et dans sa hauteur, surtout en rapport avec la modification de la densité et de la distribution des classes de diamètre, le tout dépendant à la fois de l'évolution naturelle et des opérations sylvicoles.

Rappelons enfin, que nous considérons une seule qualité stationnelle (site index) pour l'ensemble du massif étudié.

\section{Résultats et discussion}

\subsection{Croissance et accroissement du diamètre}

L'examen des données sur la croissance en diamètre, à partir des figures $2 \mathrm{a}$ et $2 \mathrm{~b}$, indique que la croissance est sensiblement la même pour les deux essences dans la première classe. Cependant, il faut noter que la différenciation entre la première et la deuxième classe s'est faite plus rapidement chez l'érable à sucre, soit vers l'âge de 24 ans, ou en 1964, alors que chez le hêtre, elle est plus lente à se produire, soit vers l'âge moyen de 32 ans, ou en 1972. Quant à la différenciation entre la deuxième et la troisième classe, elle semble avoir la même durée pour les deux essences et vers l'âge de 26 ans, elle est déjà réalisée. Cette constatation est encore plus évidente après l'analyse des accroissements annuels courants des figures $2 \mathrm{c}$ et $2 \mathrm{~d}$.

En effet, on peut observer le maintien de la vitesse de croissance pour les premières classes et une diminution soutenue pour les autres classes sociales. De plus, on peut signaler, pour la deuxième classe, une meilleure productivité chez le hêtre que chez l'érable. 

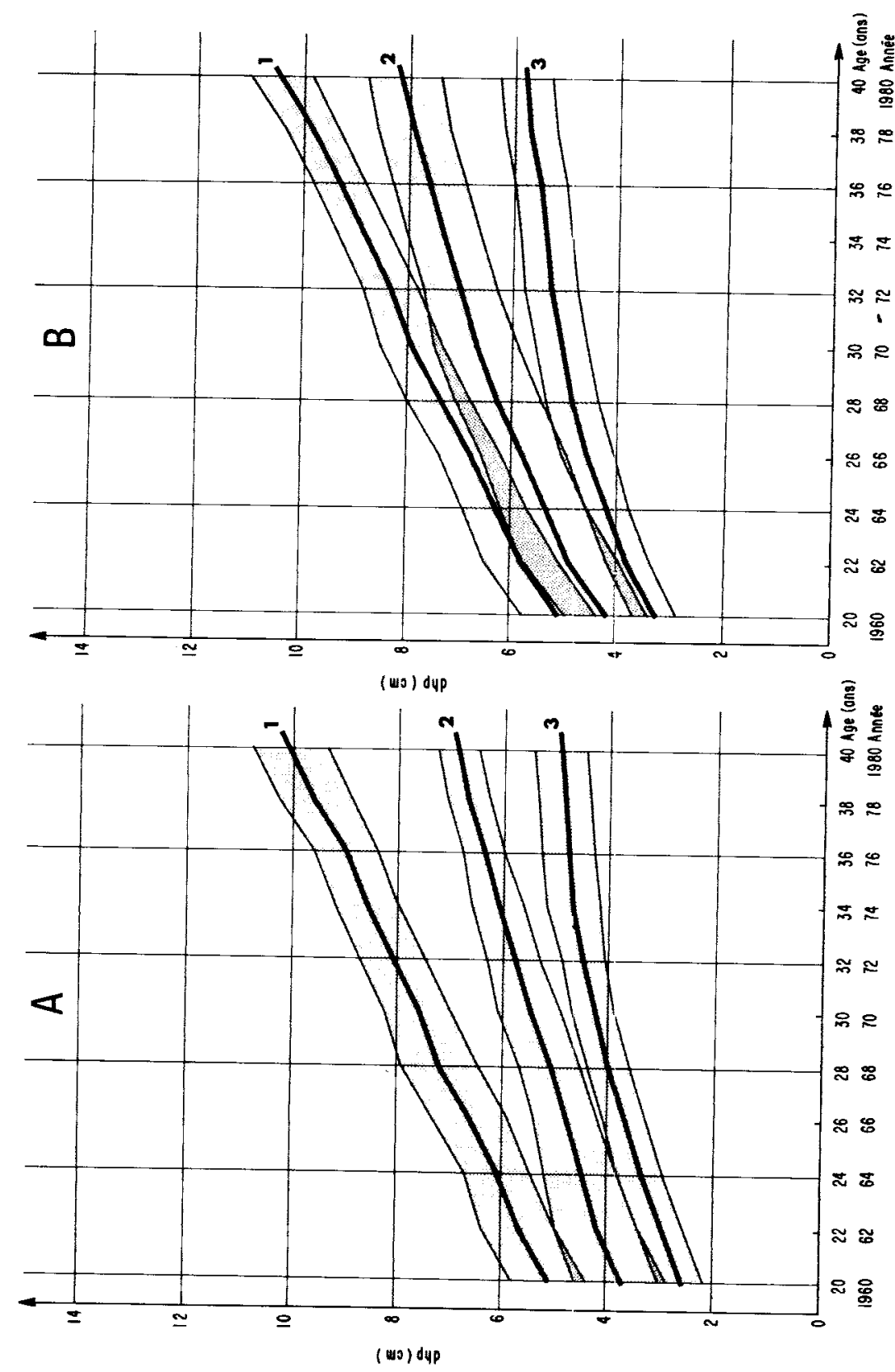

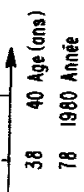

응

$\pm=$

$\approx \approx$

옹

$\approx 8$

$\stackrel{8}{\circ}$

$\approx \mathbb{5}$

$\approx \approx$

$\therefore$ 용

票

票

$\infty \approx$

$\infty$

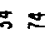

$\approx \approx$

유

:

8

\&

$\dot{8}$

$\stackrel{3}{3}$

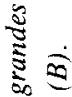

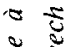

\&

$\Xi$

$\bar{T}$

सิ

$\div$ ร

$\stackrel{8}{5}$

क

है

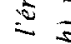

จ

इ छ

도

a

है

प)

5

芩

:

인

용

5

32

3

施

$\approx$

\&

胥 ญ

\&

竎

.

s

ڤั) 


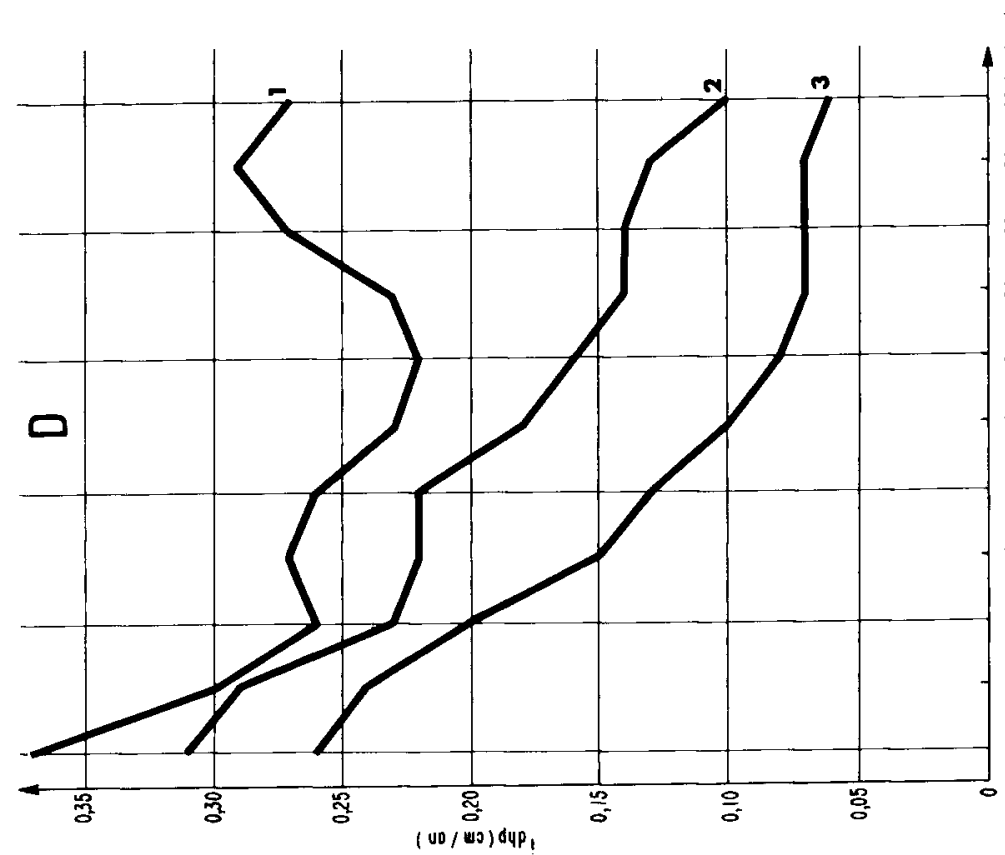

$\bar{v}$

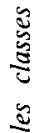

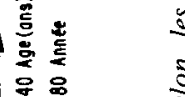

ळ

$\stackrel{\infty}{\infty}$

$\widehat{\theta}$

$\infty$

$\theta$

है

म

$\approx \approx \quad$ है

क $P$ के है

$\approx 8$ 离

$\therefore: \quad \frac{5}{3}$

¿

$\approx \approx \quad$ a

요 $\quad$ जे

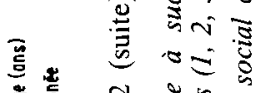

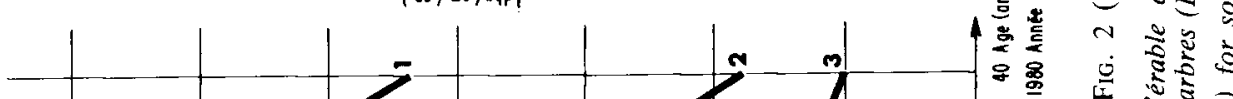

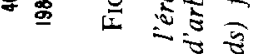

$\infty$

in:

$\lesssim \quad 0$
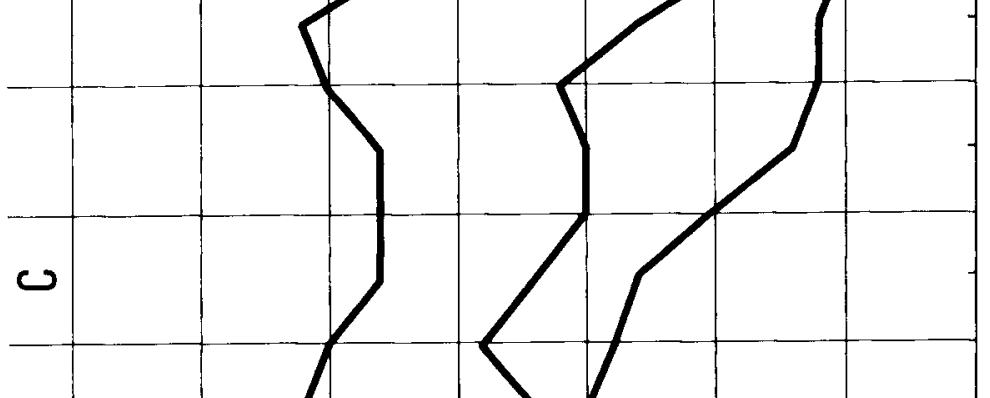

m

$\approx \approx$

$\$ 2$

$\approx \$$

$\approx$

\& $v$

₹

ป है

․

28

$\pm \pi$

ร ะ

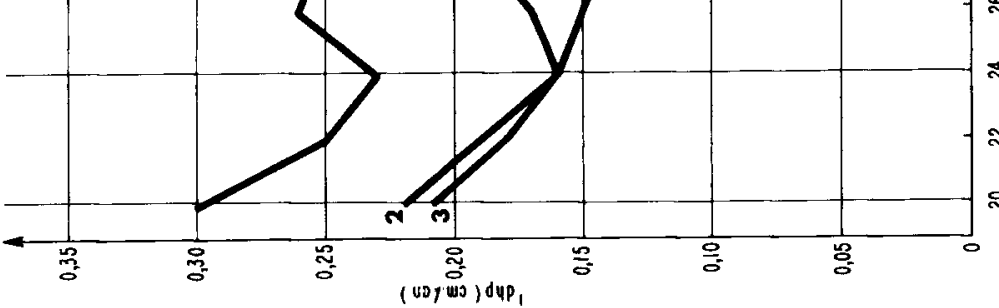

2 


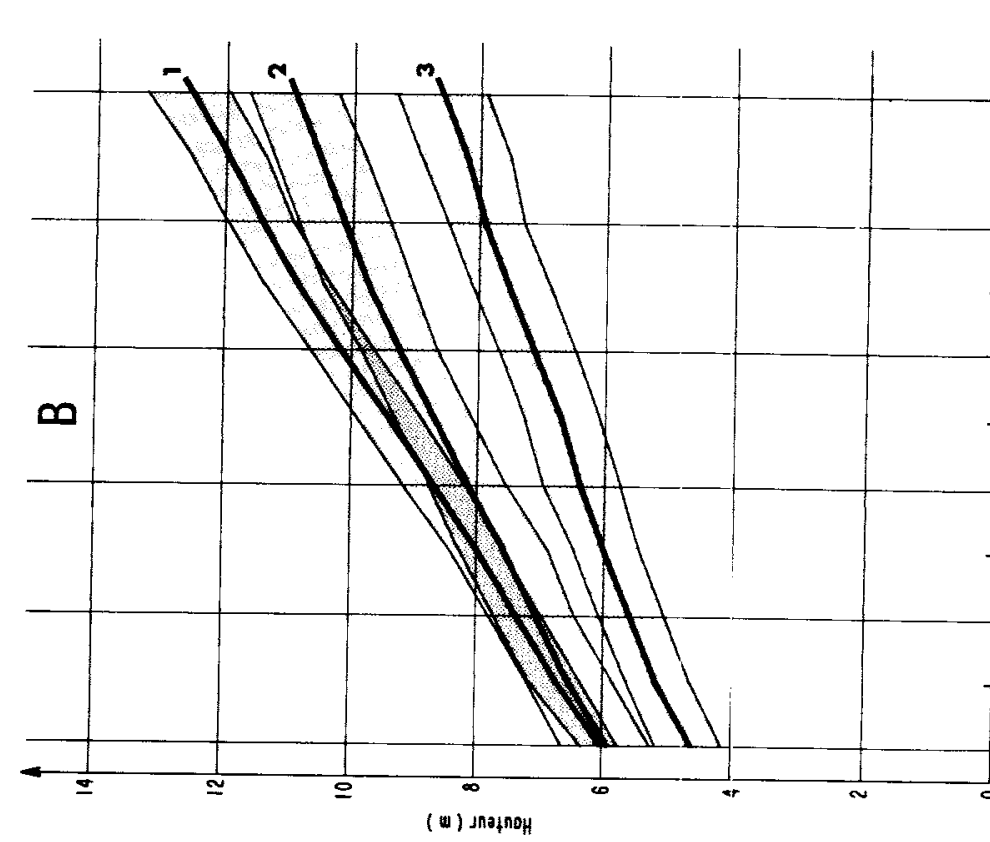

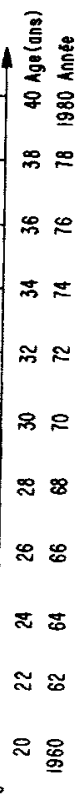

sิ

$\stackrel{3}{3}$

ฐัँ

क⿺⿻一𠃋冫

¿

ะ

전

ए

डิ

$心$

5

ฐ

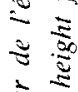

芯

产

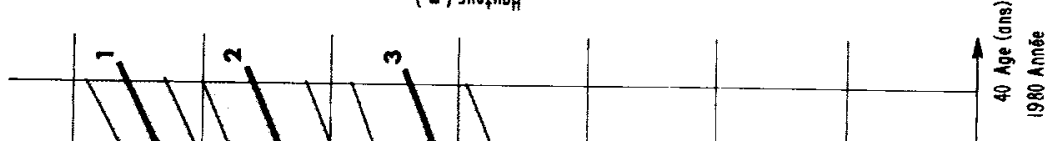

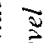

I)

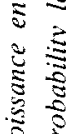

$\infty$

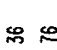

$+$

$\approx \simeq$

을

$\approx$

$\stackrel{8}{\infty}$

¿ \&

28

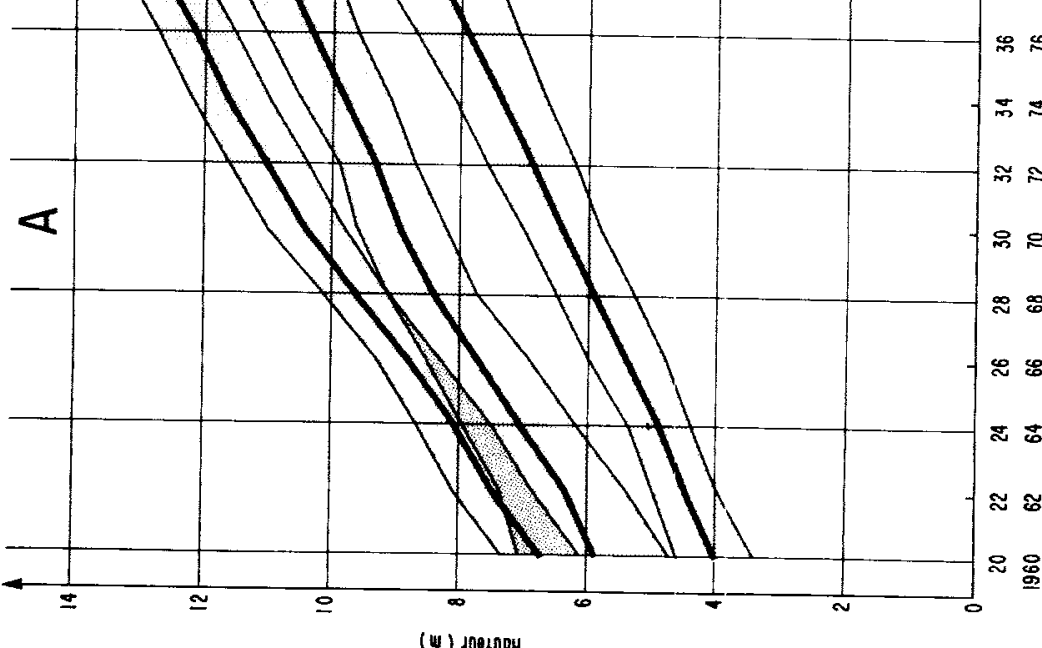

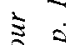

$2 \longleftarrow$

$\$$

2.

$心$

壱

$\div$

ปิ 

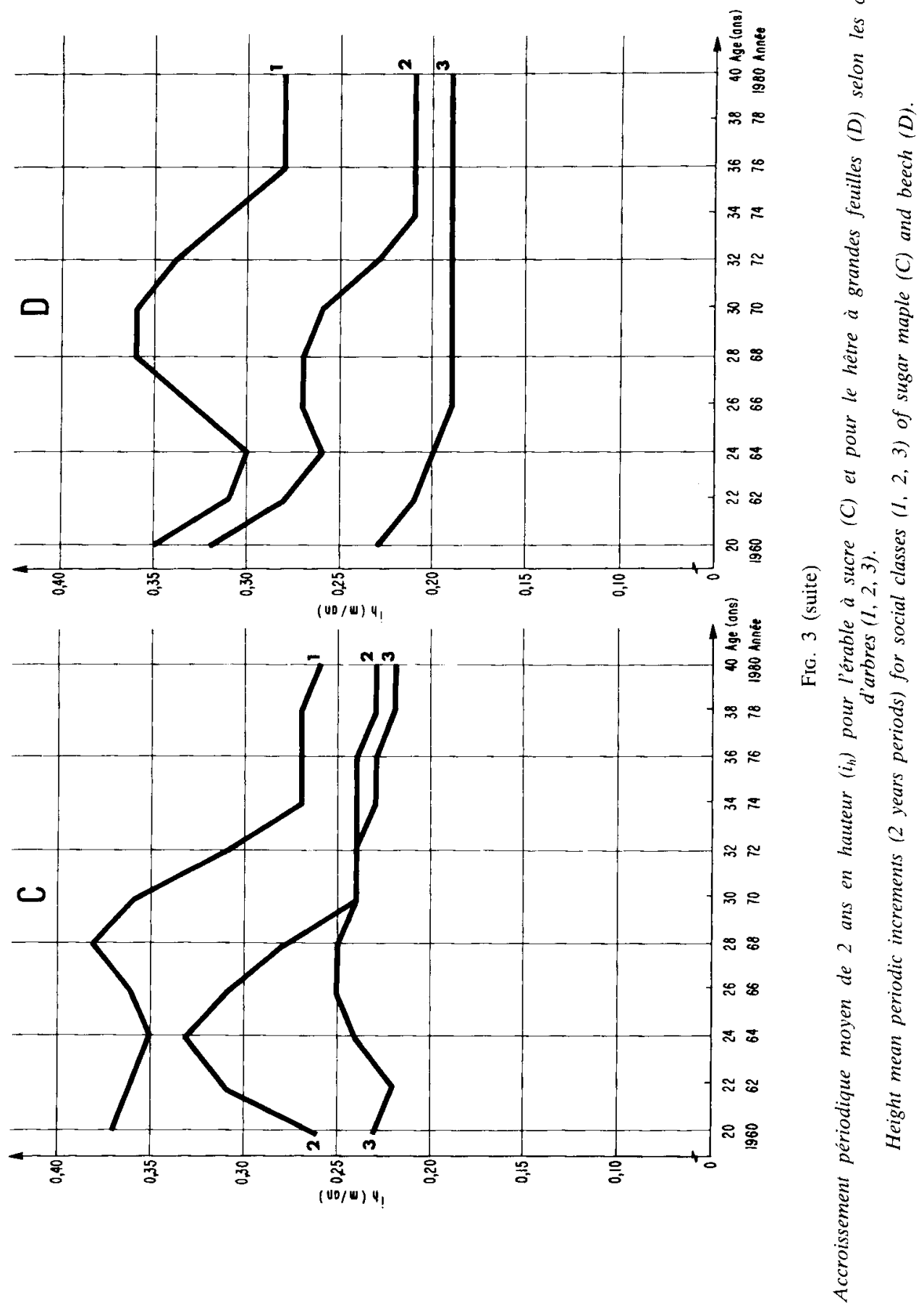

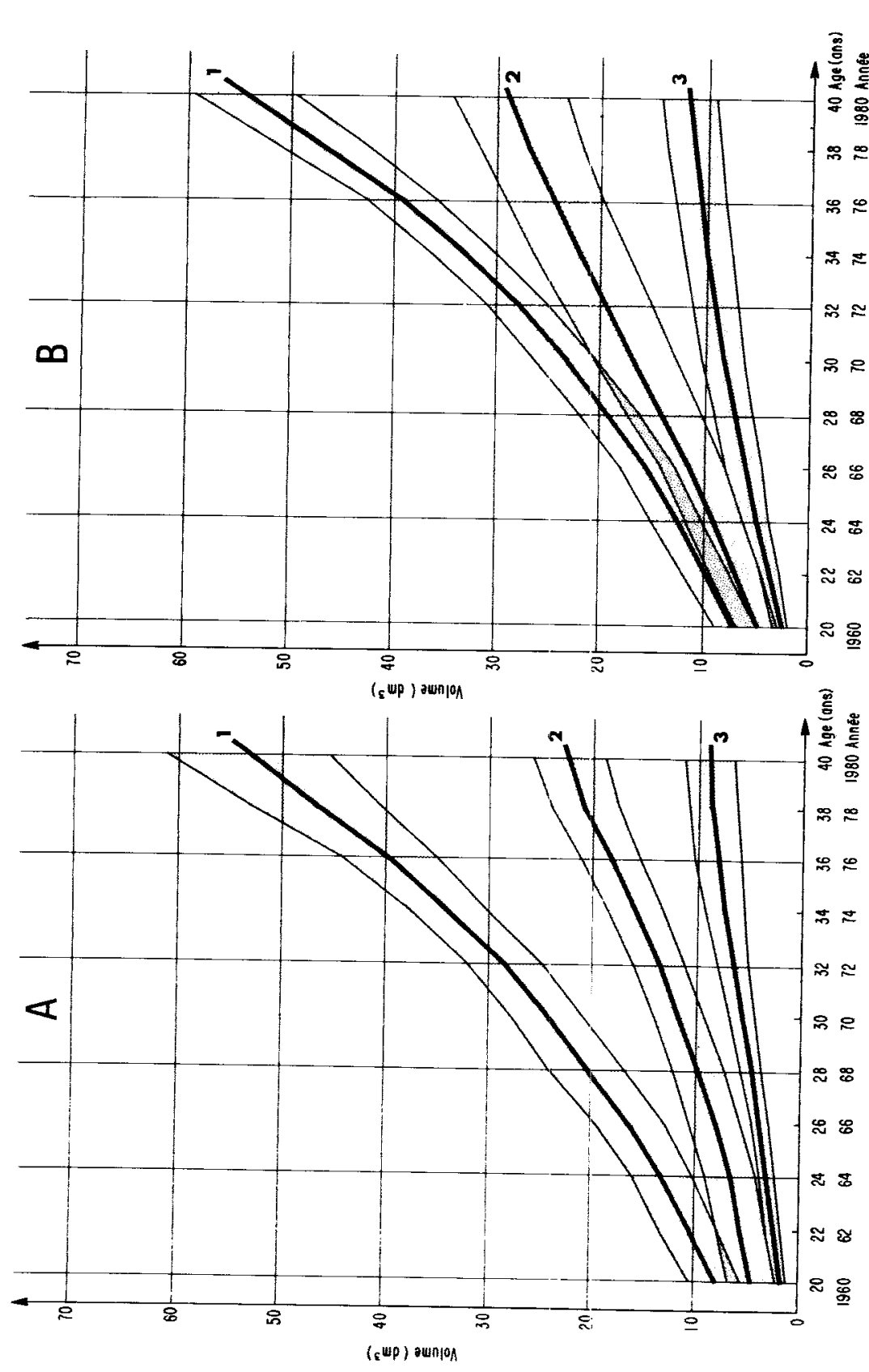

s

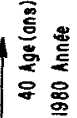

$\infty$

$\mathscr{0} \div$

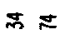

$\approx \approx$

유은

๓ 8

$\approx$

$\approx \Sigma$

$\approx \approx$

요용

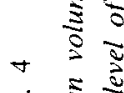

$\dot{5}$

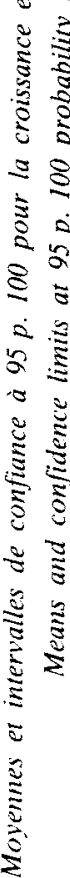



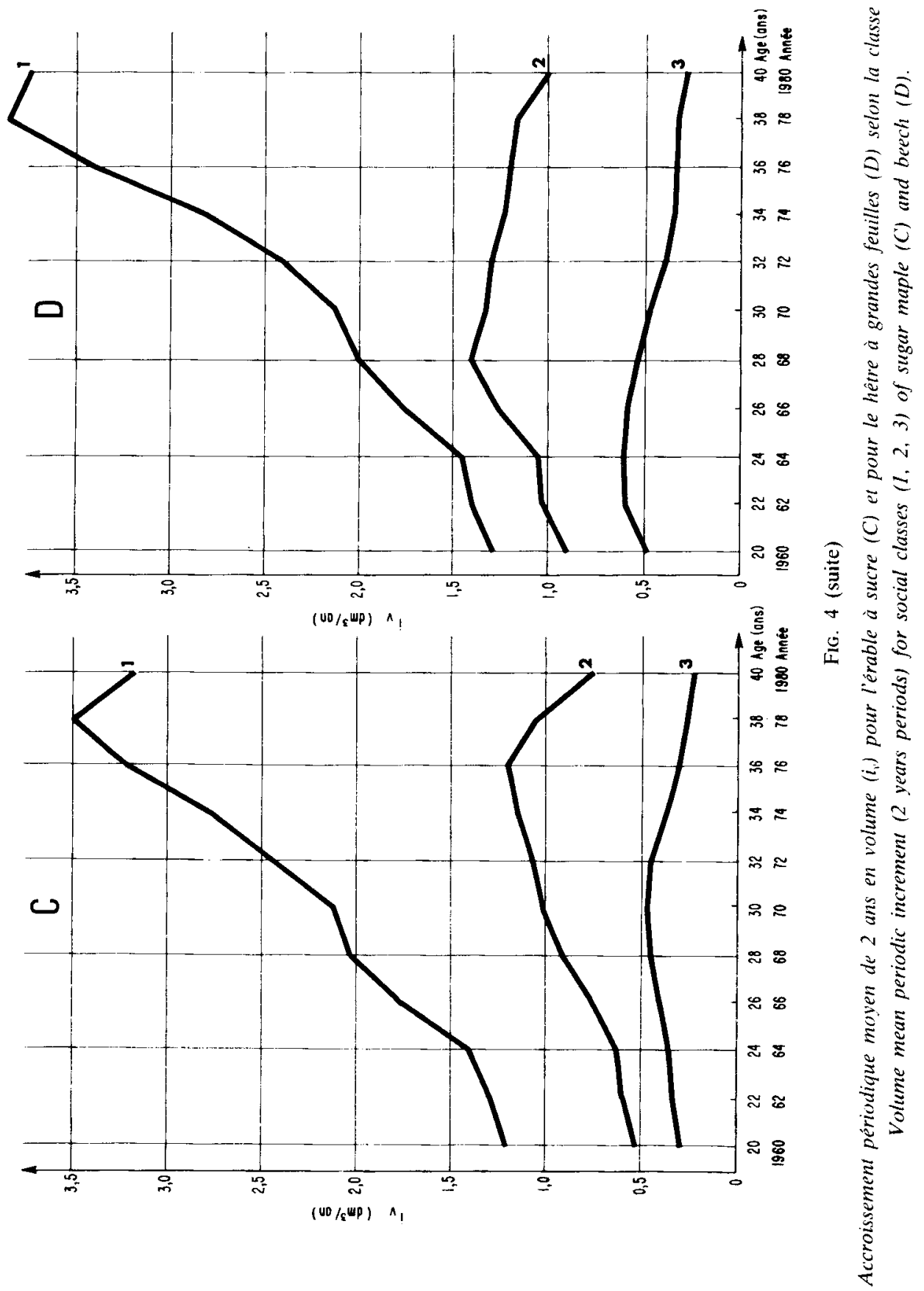


\subsection{Croissance et accroissement en hauteur}

La principale tendance observée pour l'accroissement en diamètre se vérifie à l'examen des hauteurs; selon les figures $3 \mathrm{a}$ et $3 \mathrm{~b}$, la hauteur de l'arbre moyen de troisième classe se distingue de celle de la deuxième dès l'âge de 20 ans et ceci, pour les deux essences. La distinction entre les deux classes supérieures ne se fait au plus tôt, que 10 ans plus tard. De plus, la compétition en hauteur se poursuit, bien que les différences de diamètre soient déjà significatives. Ce dernier phénomène est encore plus évident à l'examen comparatif des accroissements courants en diamètre et en hauteur ; le déclin de l'accroissement en diamètre de la troisième classe est déjà amorcé au début de la période à l'étude tandis que l'accroissement en hauteur se maintient toujours vingt ans plus tard (fig. 2c, 2d, 3c,3d). Les individus dominés, avec un potentiel de croissance plus réduit que les plus forts, sacrifieraient donc leur croissance en diamètre au profit d'une place au soleil ; ce faisant, les individus des classes inférieures acquéreraient une forme plus élancée et donc, des rapports hauteur/diamètre supérieurs à ceux de leurs dominants (tabl. 1).

Les accroissements des deux espèces sont comparables tant en hauteur qu'en diamètre indiquant qu'aucune des deux espèces ne domine l'autre dans un peuplement mélangé constitué par ces deux espèces.

\subsection{Croissance et accroissements en volume}

La croissance en volume d'un arbre, comme d'un peuplement, résulte des changements en diamètre, en hauteur et en coefficient de forme dans le temps. Pour visualiser l'effet de la position sociale sur la croissance en volume, nous avons rapporté sur les figures $4 a$ et $4 \mathbf{b}$ les intervalles de confiance qui délimitent la croissance moyenne en volume de trois classes sociales pour les deux essences. L'examen des courbes fait ressortir clairement à quel point l'effet de la position sociale est grand sur la production en volume. On peut donc dire que la hiérarchie sociale reflète des différences considérables dans la productivité des arbres au sein d'un peuplement.

D'après l'analyse des données sur la croissance en volume des deux essences, l'individualisation des classes d'arbres se produit assez tôt. En effet, la première classe se distingue de la deuxième chez l'érable à sucre vers 1963, ou à l'âge de 23 ans, c'està-dire à une hauteur dominante d'environ 7 mètres et la deuxième de la troisième classe à l'âge de 25 ans. Chez le hêtre à grandes feuilles, la première classe se distingue de la deuxième bien plus tard, soit à l'âge de 30 ans, ou à une hauteur dominante d'environ 9 mètres alors que la deuxième se démarque plus tôt de la troisième, soit à l'âge de 25 ans.

L'analyse des accroissements en volume (fig. $4 c$ et $4 d$ ) indique qu'ils sont sensiblement les mêmes entre l'érable à sucre et le hêtre à grandes feuilles par classe d'arbres pour la période étudiée. Cette même analyse montre aussi que la hiérarchie au sein du peuplement, telle qu'exprimée par la productivité cles différentes classes d'arbres semble être fixée d'une façon définitive. On peut remarquer sur ces figures que la culmination des accroissements en volume se produit d'abord dans la troisième classe, plus tard dans la deuxième et enfin, dans la première classe. Ces données nous permettent d'apprécier aussi la vigueur et la sensibilité des arbres de différentes classes. En effet, si la première classe est en pleine croissance et susceptible encore de réagir aux opéra- 
tions sylvicoles, le vieillissement accéléré des arbres de la deuxième et surtout de la troisième classe ne permet à ces derniers qu'un rôle passif de remplissage.

Pour mieux visualiser l'importance de la différence entre la productıité des principales classes d'arbres, nous avons exprimé la proportion de la deuxième et de la troisième classe en pourcentage de la première classe au tableau 2 . On peut y remarquer que ces différences sont relativement faibles dans l'accroissement en hauteur, alors qu'elles sont très prononcées dans le cas d'accroissements en diamètre et en volume. En effet, si en 1980 la production en volume de la première classe est de 100 p. 100 , celui de la deuxième est de 25 p. 100 , alors que celui de la troisième classe est de 7 p. 100 seulement par rapport à la première classe, indifféremment pour l'érable à sucre et pour le hêtre à grandes feuilles.

\section{TABLEAU 2}

Accroissements exprimés en p. 100 de la première classe d'arbres en dhp ( $\left.i_{d h p}\right)$, en hauteur $\left(i_{h}\right)$ et en volume $\left(i_{v}\right)$ pour l'érable à sucre et pour le hêtre à grandes feuilles par classe d'arbres $(1,2,3)$.

Breast height diameter $\left(i_{d h p}\right)$, height $\left(i_{h}\right)$ and volume $\left(i_{v}\right)$ relative increments of sugar maple and beech; (increment of class $I$ is $100 \mathrm{p}$. cent).

\begin{tabular}{|c|c|c|c|c|c|c|c|c|c|c|c|}
\hline \multirow{3}{*}{ Essence } & \multirow{3}{*}{ Année } & \multirow{3}{*}{$\begin{array}{c}\text { Age } \\
\text { moyen } \\
(\text { ans })\end{array} \mid$} & \multirow{2}{*}{\multicolumn{3}{|c|}{ 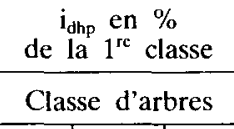 }} & \multirow{2}{*}{\multicolumn{3}{|c|}{ 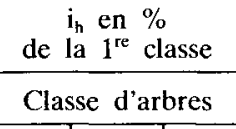 }} & \multirow{2}{*}{\multicolumn{3}{|c|}{ 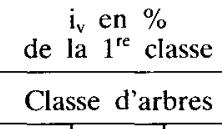 }} \\
\hline & & & & & & & & & & & \\
\hline & & & 1 & 2 & 3 & 1 & 2 & 3 & 1 & 2 & 3 \\
\hline \multirow{11}{*}{ Erable à sucre } & 1960 & 20 & 100 & 73 & 70 & 100 & 70 & 62 & 100 & 43 & 24 \\
\hline & 1962 & 22 & 100 & 76 & 72 & 100 & 86 & 61 & 100 & 46 & 26 \\
\hline & 1964 & 24 & 100 & 70 & 74 & 100 & 94 & 69 & 100 & 44 & 26 \\
\hline & 1966 & 26 & 100 & 65 & 58 & 100 & 86 & 69 & 100 & 42 & 23 \\
\hline & 1968 & 28 & 100 & 76 & 56 & 100 & 74 & 66 & 100 & 45 & 22 \\
\hline & 1970 & 30 & 100 & 74 & 51 & 100 & 67 & 67 & 100 & 48 & 22 \\
\hline & 1972 & 32 & 100 & 65 & 43 & 100 & 77 & 77 & 100 & 44 & 18 \\
\hline & 1974 & 34 & 100 & 65 & 30 & 100 & 89 & 85 & 100 & 41 & 14 \\
\hline & 1976 & 36 & 100 & 64 & 24 & 100 & 89 & 85 & 100 & 38 & 10 \\
\hline & 1978 & 38 & 100 & 50 & 23 & 100 & 85 & 81 & 100 & 31 & 7 \\
\hline & 1980 & 40 & 100 & 41 & 23 & 100 & 88 & 85 & 100 & 24 & 7 \\
\hline \multirow{11}{*}{$\begin{array}{l}\text { Hêtre à grandes } \\
\text { feuilles }\end{array}$} & 1960 & 20 & 100 & 84 & 70 & 100 & 91 & 66 & 100 & 71 & 24 \\
\hline & 1962 & 22 & 100 & 97 & 80 & 100 & 87 & 68 & 100 & 74 & 26 \\
\hline & 1964 & 24 & 100 & 88 & 77 & 100 & 90 & 67 & 100 & 73 & 26 \\
\hline & 1966 & 26 & 100 & 81 & 56 & 100 & 82 & 58 & 100 & 73 & 23 \\
\hline & 1968 & 28 & 100 & 85 & 50 & 100 & 75 & 53 & 100 & 70 & 22 \\
\hline & 1970 & 30 & 100 & 78 & 43 & 100 & 72 & 53 & 100 & 63 & 22 \\
\hline & 1972 & 32 & 100 & 73 & 36 & 100 & 68 & 56 & 100 & 54 & 18 \\
\hline & 1974 & 34 & 100 & 61 & 30 & 100 & 68 & 61 & 100 & 44 & 14 \\
\hline & 1976 & 36 & 100 & 52 & 26 & 100 & 75 & 68 & 100 & 36 & 10 \\
\hline & 1978 & 38 & 100 & 45 & 24 & 100 & 75 & 68 & 100 & 30 & 7 \\
\hline & 1980 & 40 & 100 & 37 & 22 & 100 & 75 & 68 & 100 & 26 & 7 \\
\hline
\end{tabular}


Ces résultats démontrent à quel point il est nécessaire de connaître la capacité de production des arbres, afin d'être en mesure de favoriser les individus avec le meilleur potentiel de croissance.

Cependant, pour le praticien à cause de l'impossibilité de mesurer l'accroissement en volume directement sur le terrain, il faut considérer une autre grandeur dendrométrique toute aussi indicatrice du potentiel de croissance. Selon les résultats du tableau 2, la meilleure grandeur pour le faire est l'accroissement en diamètre. Grâce à sa mesure directe sur le terrain, l'accroissement en diamètre permet au forestier d'apprécier le potentiel de la croissance des arbres et d'établir leur classification fonctionnelle.

\section{Conclusion}

L'étude de la croissance et du développement de l'érable à sucre et du hêtre à grandes feuilles fait ressortir les différences très importantes entre les classes d'arbres. En effet, la différenciation sociale, déjà amorcée à l'âge de 20 ans, s'accentue de plus en plus avec l'âge pour être fixée dans les dix années suivantes. Les plus faibles individus activent leur accroissement en hauteur au détriment du diamètre. En s'épuisant ainsi rapidement, ils sont éliminés de la course.

La position sociale de l'arbre se reflète surtout dans la vigueur de la croissance et dans la culmination de l'accroissement courant. Selon les résultats obtenus, la grandeur dendrométrique la plus sensible à la position sociale dans le peuplement est le volume de l'arbre et en particulier son accroissement annuel courant. Mesure de la productivité, cet accroissement est un bon indicateur de la position sociale de l'arbre dans le peuplement. L'examen des données des deux essences montre qu'à la fin de la période de comparaison, l'accroissement de la première classe est 4 fois plus grand que celui de la deuxième et 14 fois plus grand que celui de lá troisième classe.

Cependant, le diamètre (ou la grosseur relative) de l'arbre représente la grandeur directement mesurable la plus représentative de la classe sociale.

Quant au comportement spécifique, rien n’indique pour le moment qu'une des deux espèces dominera éventuellement lautre puisque les derniers accroissements courants restent comparables.

Compte tenu de son importance, la notion de position mériterait d'être davantage précisée. D'abord, les niveaux de production des individus pourraient être définis par des dimensions de cime absolues ou relatives telles que celles proposées par l'IUFRO (SchoвER, 1967). On compléterait aussi l'image de la différenciation sociale au sein d'un peuplement en établissant la proportion d'individus de chaque classe sociale pour différentes époques de la vie d'un peuplement. Enfin, l'effet des éclaircies sur la différenciation en classes sociales ne sera connue qu'en observant les distributions des arbres selon la position sociale dans les peuplements traités. 


\author{
Summary \\ Growth of sugar maple and beech according \\ to social classes of the stand
}

Following an experimental thinning, ¿o sugar maples and 33 beeches were sampled and divided into three social classe?. The study was carried out in a mixed hardwood seedling stand in the Temiscouna-Restigouche section (L.6, Rowe, 1972) of the Quebec province in Canada. Diameter, height and volume growth measured by stem analysis are described here. Mean ages for the three classes defined are comprised between 40 and 43 years at the time of thinning, but significant differences in diameter and height already exist. In fact, differentiation of trees into classes (and therefore stand hierarchy) are fixed after 30 years, i.e. when the stand reaches a dominant height of 10 meters. Height competition, mostly between the first 2 classes, still exists long after the best classes dominate the weakest ones in diameter or volume growth. Relative increments are expressed as ratios of the first class for the lowest two classes. It can be noticed that, while relative height increment for class three still has a value of at least $68 \mathrm{p}$. 100 , relative diameter and volume increments are as low as 22 and 7 p. 100 respectively. Consequently, the best dendrometrical indicator found for expressing the social class of a tree is volume. Relative diameter of lower classes (two and three) decreases more than relative height, probably as a result of competition for light. Therefore, diameter is more appropriate than height for practical differentiation of trees social classes. Height and diameter increments for the first classes of the two species are similar from 20 to 40 years of age. Volume growth for both species is also comparable up to 40 years.

Further studies are proposed to relate yield classes to crown dimensions, to establish relative importance of the 3 classes at different ages of the stands, and to study distribution of trees in thinned stands.

Key words: Tree classes, stem analysis, Acer saccharum, Fagus grandifolia.

\title{
Références bibliographiques
}

Assmann E., 1961. Waldertragskunde, BLV Munchen, $490 \mathrm{p}$.

Delvaux J., 1981. Différenciation sociale. Schweiz. Z. Fostwes., 132 (9), 733-749.

DvoreckiJ M.L., 1966. O stepeni ustojcivosti srednich derevev drevostoja s vozrastom. Lesnoj zurnal, 9, 6-9.

Grandtner M.M. La végétation forestière du Québec méridional. Presses Univ. Laval, Québec, $216 \mathrm{p}$.

Rowf J.S., 1972. Forest regions of Canada. Department of the Environment. Can. For. Serv., publication no. 1300 .

Schober R., 1967. Ideas and proposals for a new system of thinning and tree classification. Proc. 14th Congr. Int. Union of For. Res. Organ., Münich, Pt. VI, Sect. 25, 61-95.

Schütz J.Ph., 1981. L'éclaircie sélective de Schädelin, évolution et pratique actuelle. R.F.F., XXXIII-no sp., 7-18.

Smelko S., 1976. Zakonitosti rastu a prirastku stromov $v$ poraste odvodene $z$ kmenovych analyz. Priroda, 18 (1), 59-77.

Villeneuve G.O., 1959. Bref aperçu climatique du Québec méridional. Cahier de géogr, Québec, 3, $153-162$.

Wilson C., 1971. Le climat du Québec. Première partie : atlas climatique. Canada, Serv. météo., Etudes climatologiques II.

Zarnovican R., 1985. Analyse de tige : une méthode à redécouvrir. Nat. can., 112, 253-260. 